\title{
Real-Time Density Estimation in Urban Environments by using Vehicular Communications
}

\author{
Julio A. Sanguesa, Manuel Fogue, Piedad Garrido, \\ Francisco J. Martinez \\ Computer Science and System Engineering Department \\ University of Zaragoza, Spain \\ Email: \{jsanguesa, mfogue, piedad, f.martinez\}@unizar.es
}

\author{
Juan-Carlos Cano, Carlos T. Calafate, Pietro Manzoni, \\ Computer Engineering Department \\ Universitat Politècnica de València, Spain \\ Email: $\{$ jucano, calafate, pmanzoni\}@disca.upv.es
}

\begin{abstract}
Knowing the density of vehicles in a vehicular communications environment is important, as better opportunities for wireless communication can show up. This paper studies the importance of predicting the density of vehicles in vehicular environments to take decisions for enhancing the dissemination of warning messages between vehicles. Moreover, we propose a mechanism which allows the estimation of the vehicular density within a certain urban environment, using as parameters the number of beacons received per vehicle, and the topological characteristics of the environment where the vehicles are located.
\end{abstract}

Index Terms-vehicular networks, vehicular density estimation, warning message dissemination, VANETs.

\section{INTRODUCTION}

Vehicular Networks (VNs) are wireless communication networks that support cooperative driving among communicating cars on the road. Vehicles act as communication nodes and relays, forming dynamic vehicular networks together with other near-by vehicles on the road. VNs involve vehicleto-vehicle (V2V) [1] and vehicle-to-infrastructure (V2I) [2] communications, and have received a greater attention in the recent years.

The specific characteristics of vehicular networks favor the development of attractive and challenging services and applications. However, most of these applications could be more efficient if the protocols involved become aware of the density of vehicles at any given time [3], and adapt their behavior according to this factor. Thus, knowing the density of a vehicular environment is important since it allows using the wireless channel more efficiently.

One issue to keep in mind when making any proposal related to vehicular networks is to study in detail how it behaves when modifying all the possible factors [4]. However, this can be a very time-consuming task, so it is recommended to focus only on the most important factors, overlooking the rest of parameters. In [5], we studied these factors in realistic urban environments, concluding that the most significant are: (i) the density of vehicles, since messages are propagated much more easily in high density scenarios than in scenarios with a low density of vehicles (where messages cannot exploit the inherent multihop capabilities of VNs), and (ii) the urban topology, as the presence of buildings greatly affects the wireless signal propagation.
Traditionally, in Transportation Systems, vehicle density has been one of the main metrics used for assessing the road traffic conditions. A high vehicle density usually indicates that the traffic is congested. Currently, most of the vehicle density estimation approaches are designed for using infrastructure-based traffic information systems. Hence, these approaches require the deployment of vehicle detecting devices such as inductive loop detectors or traffic surveillance cameras. Consequently, these approaches do not exploit the capabilities offered by emerging self-organizing vehicular traffic information systems, where vehicles are able to collect and process the traffic information without relying on any fixed infrastructure.

In this paper we study the importance of vehicle density awareness in urban environments, and we present a solution to estimate the density of vehicles that is specially designed for Vehicular Networks. Our solution allows vehicles to continuously estimate the vehicular density in their neighborhood by accounting for the number of beacons received per vehicle, as well as the roadmap topology.

The paper is organized as follows: Section II presents in detail the parameters we used in our simulations. In Section III we discuss the importance of the vehicle density and the influence of the topology on the performance of vehicular communications. Later, in Section IV, we present in detail our proposal for real-time estimation of vehicular density, focusing on the methodology followed. In addition to assess the goodness of our proposal, we discuss the obtained results and measure the estimated error. In Section $\mathrm{V}$ we review previous works closely related to our proposal. Finally, in Section VI we present the conclusions.

\section{Simulation EnVironment}

The simulation results shown in this article have been obtained using the ns-2 simulator [6], modified to consider the IEEE $802.11 \mathrm{p}$ standard $^{1}$.

We tested our model by evaluating the performance of a Warning Message Dissemination mechanism, where each vehicle periodically broadcasts information about itself or about an abnormal situation (icy roads, traffic jam, etc.).

${ }^{1}$ All these improvements and modifications are available in http://www.grc.upv.es/software/ 


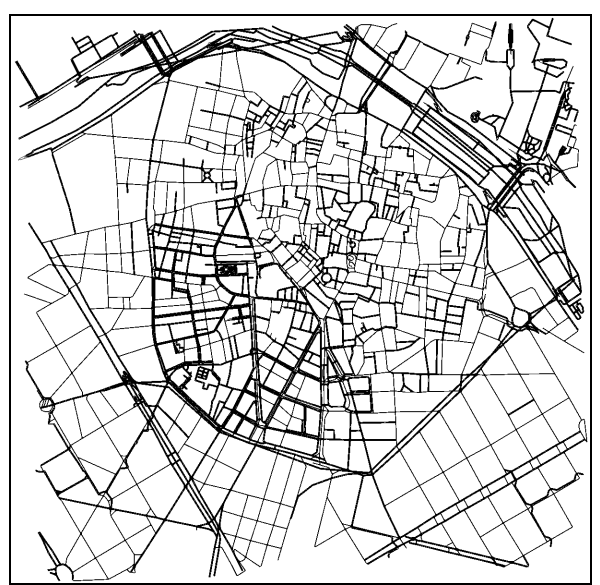

(a)

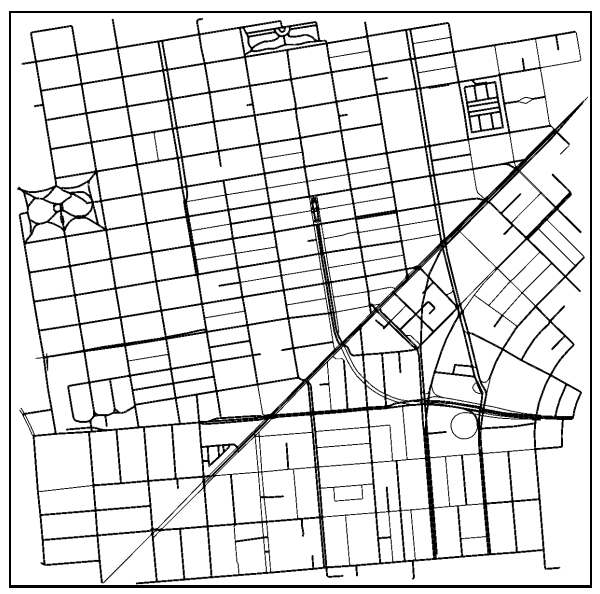

(b)

Fig. 1. Scenarios used in our simulations: (a) fragment of the city of Valencia (Spain), and (b) fragment of the city of San Francisco (USA).

In order to mitigate the broadcast storm problem [7], our simulations use the enhanced Message Dissemination based on Roadmaps (eMDR) scheme [8]. The eMDR scheme only allows forwarding messages when the distance between sender and receiver is greater than a threshold, or in situations where the receiver is the closest vehicle to a junction, and rebroadcasting could allow the message to reach new streets.

To prove how maps affect the performance of vehicular communications, we selected two street maps, each one representing $4 \mathrm{~km}^{2}$ of square area. Figure 1 shows the topology of the maps used in the simulations. As shown, Valencia is an example of a city with a high density of streets and junctions, arranged in a complex layout that differs from typical Manhattan-grid layouts, while San Francisco has a very regular street layout with a lower number of streets and junctions. As for the mobility of the vehicles, it has been performed with CityMob for Roadmaps (C4R) [11], a mobility generator able to import maps directly from OpenStreetMap
TABLE I

PARAMETERS USED FOR THE SIMULATIONS

\begin{tabular}{|l|c|}
\hline Parameter & Value \\
\hline roadmaps & Valencia and San Francisco \\
number of vehicles & {$[100,200,300 \ldots 1000]$} \\
number of collided vehicles & 3 \\
roadmap size & $2000 \mathrm{~m} \times 2000 \mathrm{~m}$ \\
warning message size & $256 B$ \\
beacon message size & $512 B$ \\
warning messages priority & $A C 3$ \\
beacon priority & $A C 0$ \\
interval between messages & 1 second \\
MAC/PHY & $802.11 \mathrm{p}$ \\
radio propagation model & $R A V[9]$ \\
mobility model & Krauss $[10]$ \\
channel bandwidth & $6 M b p s$ \\
max. transmission range & $400 \mathrm{~m}$ \\
\hline
\end{tabular}

[12]. Table I shows the parameters used for the simulations.

We selected three metrics to help us evaluate the performance of warning dissemination processes in a VN: (i) the warning notification time, (ii) the percentage of vehicles informed, and (iii) the number of messages received per vehicle. The warning notification time is the time required by normal vehicles to receive a warning message sent by a warning-mode vehicle, and it is critical when dealing with the usefulness of the system (a warning message delivered too late is useless when facing dangerous situations). The percentage of vehicles informed is the percentage of vehicles that have already received these warning messages. Finally, the number of messages received per vehicle (including beacons and warning messages) provides an indication of the level of channel contention.

All the results represent an average of over 50 repetitions with different scenarios (maximum error of $10 \%$ with a degree of confidence of $90 \%$ ), and each simulation run lasted for 180 seconds.

\section{IMPORTANCE OF THE DENSITY AND THE TOPOLOGY IN VEHICULAR NETWORKS}

In this section we study the influence that the density of vehicles and the roadmap topology have in vehicular networks, demonstrating that they are two factors that strongly affect vehicular communications performance. In this work, we focus on vehicular environments, where vehicles periodically exchange messages (beacons) that contain information about their position, speed, route, etc.; if an accident happens, the vehicles involved broadcast warning messages to nearby vehicles, and these messages are also disseminated to the rest of vehicles by using multi-hop communications.

Figure 2 shows the simulation results when varying the number of vehicles, in the scenarios of Valencia and San Francisco, respectively. We have tested with different numbers of vehicles, ranging from 100 to 1000 (i.e. from 25 to 250 vehicles $/ \mathrm{km}^{2}$ ).

As shown, in both cases, the warning notification time decreases with increasing vehicle density. For example, in the scenario of Valencia, $50 \%$ of the vehicles are informed in 20 seconds when simulating 300 vehicles, but the system 


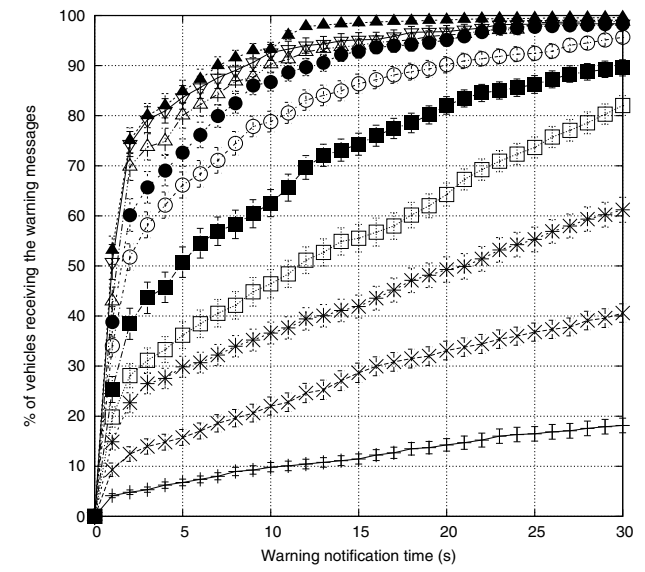

(a)

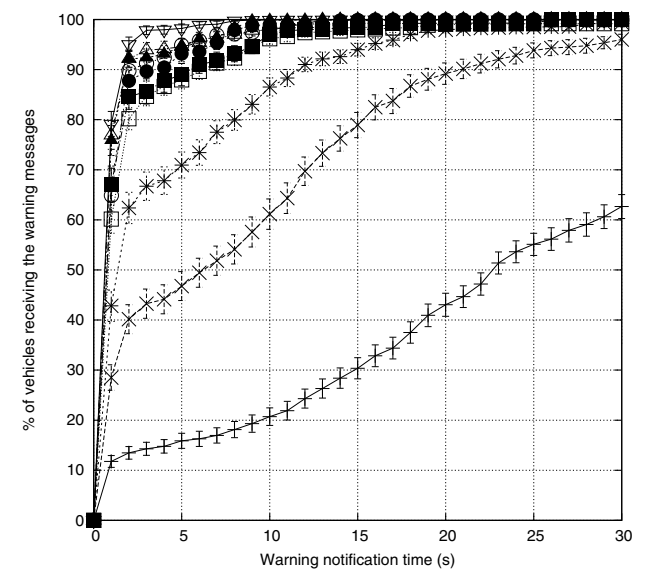

(b)

Fig. 2. Warning notification time and percentage of vehicles informed when varying the density of vehicles in: (a) Valencia (Spain), and (b) San Francisco (USA).

requires less than 5 seconds to inform the same percentage of vehicles when simulating 500 vehicles. This time is extremely reduced in very high densities (e.g., $50 \%$ of the vehicles are informed in only 1 second when simulating 1000 vehicles). This characteristic is explained because the flooding propagation of warning messages works better with higher vehicle densities. Nevertheless, highly congested scenarios can negatively affect the warning dissemination performance. As shown in Figure $2 \mathrm{a}$, the system requires less time to inform $90 \%$ of the vehicles when simulating 900 vehicles than when simulating 1000 vehicles ( 7 seconds and 9 seconds, respectively), due to excessive channel contention and collisions.

However, when simulating the scenario of San Francisco, the density of vehicles is not as determinant as in Valencia, since the warning notification time is very similar when the density of vehicles is greater or equal than 400 vehicles. In that case, more than $80 \%$ of the vehicles are correctly informed in less than 2 seconds. This characteristic is explained because the information is more easily disseminated in simpler scenarios (i.e., scenarios that have a small number of streets and junctions).

The behavior in terms of percentage of vehicles informed also depends strongly on both factors. When simulating the same number of vehicles, more vehicles are informed in San Francisco than in Valencia. As mentioned before, warning messages are more easily propagated in simpler scenarios where buildings do not affect signal propagation to the same extent than in complex roadmaps. In addition, when the density of vehicles increases, the percentage of vehicles informed also increases, since the probability of successful communications increases in higher density scenarios. Only in very high density scenarios (700 vehicles or above) do both maps present similar results in terms of vehicles informed.

Regarding the roadmap topology, Figure 3 shows the warning notification time when varying the roadmap, while main- taining fixed the number of vehicles (400 vehicles in this case). As shown, the warning notification time is lower when simulating the map of San Francisco. Information reaches about $60 \%$ of the vehicles in only 1 second, and the percentage of vehicles receiving the warning vehicles reaches $80 \%$ in only 2 seconds. In contrast, when simulating the map of Valencia, information needs more time (about 29 seconds) to reach the same percentage of vehicles. So, when the topology of the map is more complex, the percentage of vehicles informed decreases, and more time is needed to reach the same percentage of vehicles.

Concerning the number of messages received per vehicle, as expected, this number highly increases when increasing the vehicle density, although the number of messages received per vehicle highly differs depending on the map. As shown in Figure 4 , the number of messages received is considerably lower for Valencia compared to San Francisco, since the wireless signal encounters more restrictions in this roadmap, and it is usually blocked by the presence of buildings.

\section{Real-Time Vehicular Density Estimation}

The main objective of this paper is to propose a mechanism which allows estimating the density of vehicles in a specific area by using VNs. In particular, we intend to estimate the density taking into account the number of beacons received and the characteristics of the topology of the selected area (which could be obtained from the in-vehicle GPS unit). Hence, if vehicles are able to precisely estimate the vehicular density in their neighborhood, they could adequate their diffusion scheme according to this density.

To propose a method able to accurately estimate the density of vehicles, based on the number of beacons received and the roadmap topology, we made a total of 4000 experiments. These experiments involved the simulation of controlled scenarios (i.e., scenarios where the actual density is known). According to the results obtained, and using a regression 


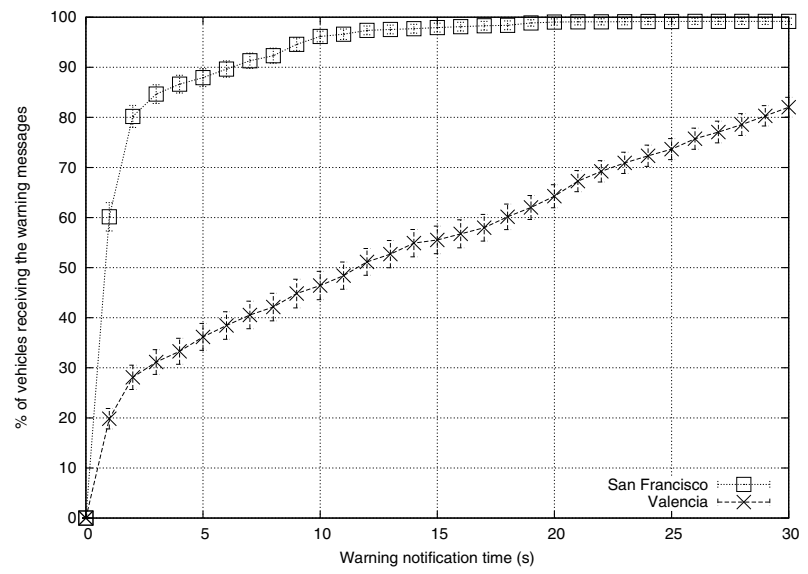

Fig. 3. Warning notification time and percentage of vehicles informed when varying the roadmap (simulating 400 vehicles).

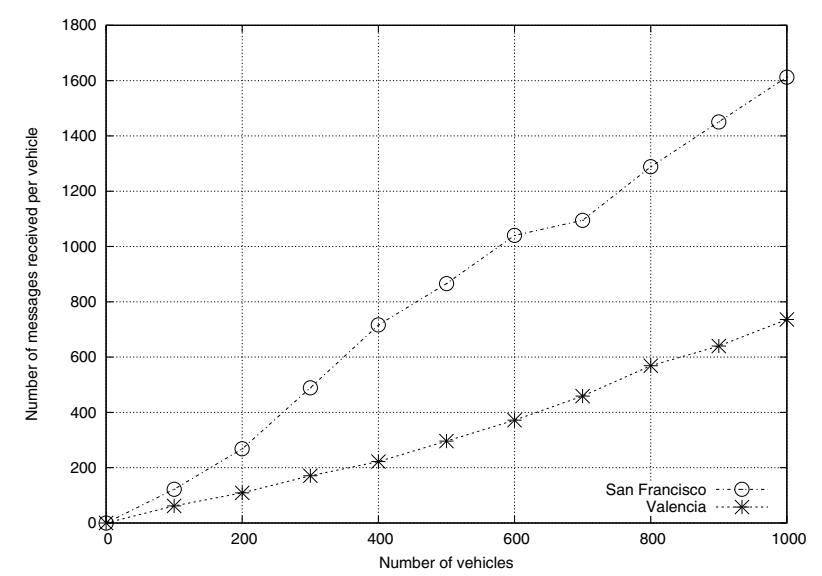

Fig. 4. Messages received per vehicle during the first 30 seconds when varying the density of the vehicles and the roadmap.

analysis, we propose an density estimation function capable of estimating the vehicular density in every urban environment at any instant of time.

The roadmaps used during the experiments to achieve the density estimation were selected in order to have different profile scenarios (i.e., with different topology characteristics). Compared to the previous section, we added 6 new cities (Rome, Rio de Janeiro, Sydney, Amsterdam, Madrid, and Los Angeles).

\section{A. Features of the Cities Studied}

The first step before starting the simulations was to obtain the main features of each roadmap (i.e., the number of streets and junctions). However, we realized that different alternatives could be selected to obtain the number of streets of a given roadmap. Basically, they are: (i) the number of streets obtained in SUMO [13], where each segment between two junctions represents a street, (ii) the number of streets obtained in
TABLE II

NUMBER OF STREETS OBTAINED DEPENDING ON THE APPROACH USED

\begin{tabular}{|c|c|c|c|}
\hline City & SUMO & OSM & RAV \\
\hline Rome & 2780 & 1484 & 1656 \\
\hline Rio De Janeiro & 758 & 377 & 542 \\
\hline Madrid & 3022 & 796 & 1495 \\
\hline Amsterdam & 1387 & 1029 & 628 \\
\hline
\end{tabular}

TABLE III

MAP FEATURES

\begin{tabular}{|c|c|c|c|}
\hline Map & Streets & Junctions & SJ Ratio \\
\hline Rome & 1656 & 1193 & 1.3880 \\
\hline Rio De Janeiro & 542 & 401 & 1.3516 \\
\hline Valencia & $\mathbf{2 8 3 1}$ & $\mathbf{2 2 3 3}$ & $\mathbf{1 . 2 6 7 8}$ \\
\hline Sydney & 872 & 814 & 1.0712 \\
\hline Amsterdam & 1494 & 1449 & 1.0310 \\
\hline \hline Madrid & 628 & 715 & 0.8783 \\
\hline San Francisco & $\mathbf{7 2 5}$ & $\mathbf{8 1 8}$ & $\mathbf{0 . 8 8 6 3}$ \\
\hline Los Angeles & 287 & 306 & 0.9379 \\
\hline
\end{tabular}

OpenStreetMap (OSM) [12], where each street has a different "name", and (iii) the number of streets according to our RAV radio propagation model, where the visibility between vehicles is considered to identify the streets [9].

Table II shows the values obtained according to each approach to count the number of streets for some of the cities studied. As shown, the differences between these approaches are significant, showing that it is important to decide which one to use in order to obtain better results. After some experiments, we realized that the third approach better correlated with the real features of cities, since the other two present some drawbacks: they are not accurate enough, or they present some errors. So, we choose this approach for the analysis that follows.

Table III shows the number of streets and junctions for each of the cities studied, obtained according to the RAV algorithm. We also added a column labeled as SJ Ratio, which represents the result of dividing the number of streets between the number of junctions. As shown, the first 5 cities (Rome, Rio, Valencia, Sydney, and Amsterdam) present a SJ ratio greater than 1, which indicates that they have a complex topology, while the rest of the cities (Madrid, San Francisco, and Los Angeles) present a lower SJ value, which indicates that they have a simple topology. Note that, although Rio de Janeiro has a relatively small number of streets and junctions, it has a complex topology since the SJ Ratio is greater than 1 .

After performing the topological analysis of the studied maps, we obtained the number of beacons received by each vehicle during 30 seconds, taking into account that each vehicle sends one beacon per second, and that these messages, unlike warning messages, are not disseminated by the rest of the vehicles.

Figure 5 shows the results obtained for the different cities studied. We also included two lines which depict the averages for each profile category (i.e., simple and complex average). As shown, two different groups can be distinguished: (i) the complex maps, which are located in the left part of the figure, and (ii) the simple maps, which are located in the right part of the figure. 


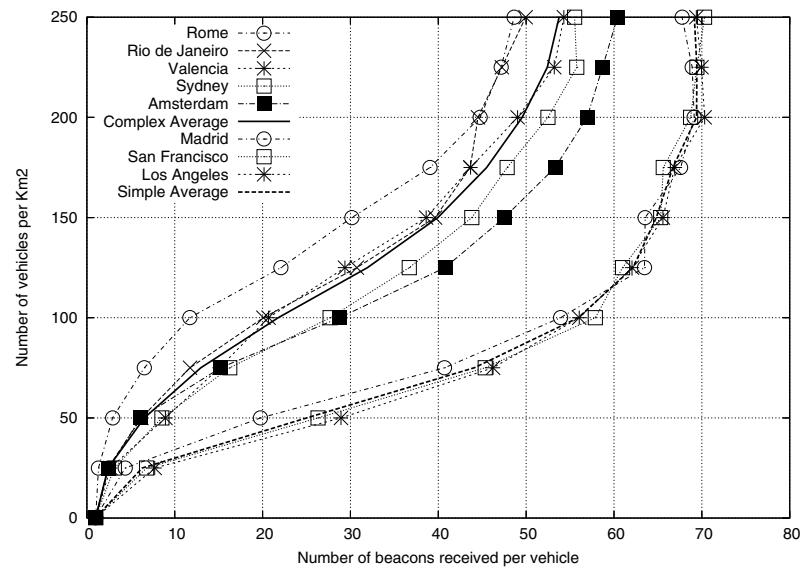

Fig. 5. Number of beacons received when varying the vehicular density.

TABLE IV

Average Percentage Difference with Respect to the Mean VALUE

\begin{tabular}{|c|c|}
\hline City & Percentage Difference \\
\hline Rome & $29.49 \%$ \\
\hline Rio De Janeiro & $5.85 \%$ \\
\hline Valencia & $\mathbf{4 . 5 6 \%}$ \\
\hline Sydney & $15.82 \%$ \\
\hline Amsterdam & $14.96 \%$ \\
\hline \hline Madrid & $6.44 \%$ \\
\hline San Francisco & $\mathbf{1 . 7 4 \%}$ \\
\hline Los Angeles & $4.70 \%$ \\
\hline
\end{tabular}

As expected, complex roadmaps present a number of beacons received lower than simple roadmaps for a similar vehicular density. In addition, we found that the simpler cities present very similar results between them, being more difficult to estimate the vehicular density in complex cities compared to simple cities. Figure 5 also shows that the vehicular density not only depends on the number of beacons received, but also on the SJ ratio (according to data shown in Table III). Therefore, the characteristics of the roadmap will be very useful in order to accurately estimate the vehicular density in a given scenario.

Table IV shows the average percentage difference with respect to the mean value. From the obtained results we observe that the cities that show a better fit for the average results are Valencia (in the complex topology group), and San Francisco (in the simple topology group). Hence, these cities could be used as reference to obtain representative results when simulating Vehicular Networks.

\section{B. Density Estimation Function}

After observing the direct relationship between the topology of the maps, the number of beacons received, and the density of vehicles, we proceed to obtain a function to estimate, with the minimum possible error, each of the curves shown in Figure 5. To this purpose, we performed a regression analysis that allowed us to find the polynomial equation offering the best fit to the data obtained through simulation.

Equation 1 shows the density estimation function, which
TABLE V

PROPOSED EQUATION COEFFICIENTS

\begin{tabular}{|c|c|}
\hline Coeff. & Value \\
\hline $\mathrm{a}$ & $-1.1138191190298828 \mathrm{E}+03$ \\
\hline $\mathrm{b}$ & $-1.0800433554686800 \mathrm{E}+01$ \\
\hline $\mathrm{c}$ & $3.1832185406821718 \mathrm{E}+03$ \\
\hline d & $-4.0336415134812398 \mathrm{E}-01$ \\
\hline f & $-3.0203454502011946 \mathrm{E}+03$ \\
\hline $\mathrm{g}$ & $2.8542014049626700 \mathrm{E}-03$ \\
\hline h & $9.5199929660347175 \mathrm{E}+02$ \\
\hline i & $3.5319225007012626 \mathrm{E}+01$ \\
\hline j & $1.6230525995036607 \mathrm{E}-01$ \\
\hline k & $-1.6615888771467137 \mathrm{E}+01$ \\
\hline
\end{tabular}

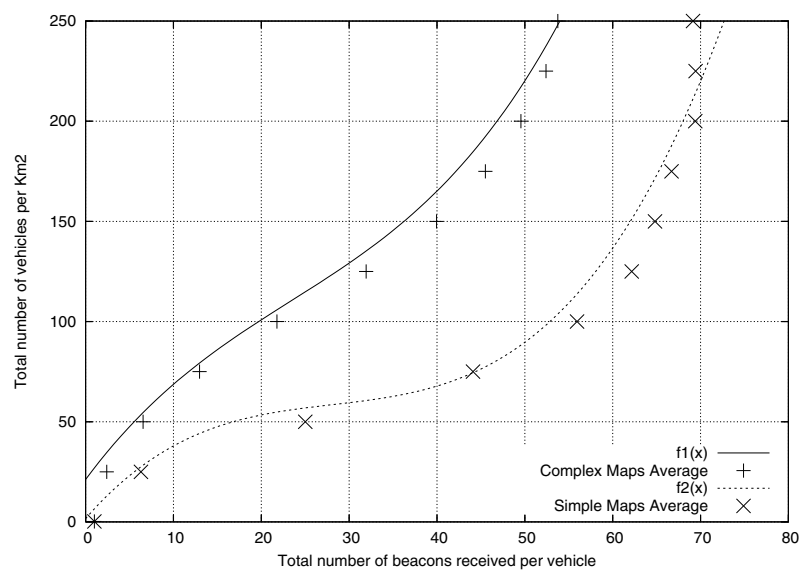

Fig. 6. Comparison between simulated and estimated average results.

is able to estimate the number of vehicles per $\mathrm{km}^{2}$ in urban scenarios, according to the number of beacons received, and the SJ ratio (i.e., streets/junctions).

$$
\begin{aligned}
f(x, y)=a+b x & +c y+d x^{2}+f y^{2}+g x^{3}+ \\
& +h y^{3}+i x y+j x^{2} y+k x y^{2}
\end{aligned}
$$

In this equation, $x$ is the number of beacons received by each vehicle, and $y$ is the SJ ratio obtained from the roadmap. The values of the polynomial coefficients $(a, b, c, d, f, g, h, i, j$, and $k)$ are listed in Table $\mathrm{V}$.

\section{Validation of our Proposal: Error Estimation}

To determine the accuracy of our proposal, it is necessary to measure the estimated error. Figure 6 shows the difference between the average values for all the cities studied, and the values obtained by our function. As shown, we achieve a very good fit for the average values obtained in the simulations. In addition, Table VI shows the different types of errors calculated when comparing our density estimation function with the values actually obtained. Note that the average relative error is of only $1.02 \%$.

\section{RELATED WORK}

Despite the importance of determining the vehicular density to improve the support for vehicular network applications, so 
TABLE VI

DENSITY ESTIMATION ERROR

\begin{tabular}{|c|c|c|}
\hline Error & Absolute & Relative \\
\hline Minimum & $-2.612027 \mathrm{E}+01$ & $-2.284800 \mathrm{E}-01$ \\
\hline Maximum & $2.169529 \mathrm{E}+01$ & $5.713108 \mathrm{E}-01$ \\
\hline Mean & $-3.176197 \mathrm{E}-10$ & $1.023340 \mathrm{E}-02$ \\
\hline Std. Error of Mean & $1.360303 \mathrm{E}+00$ & $1.714082 \mathrm{E}-02$ \\
\hline Median & $1.698901 \mathrm{E}-01$ & $-1.359121 \mathrm{E}-03$ \\
\hline
\end{tabular}

far there have not been enough studies that explored the density estimation in order to improve wireless communications in vehicular environments.

Tyagi et al. [14] considered the problem of vehicular traffic density estimation, using the information cues available in the cumulative acoustic signal acquired from a roadsideinstalled single microphone. This cumulative signal comprises several noise signals such as tire noise, engine noise, engineidling noise, occasional honks, and air turbulence noise of multiple vehicles. The occurrence and mixture weightings of these noise signals are determined by the prevalent traffic density conditions on the road segment. Using a discriminative classifier, such as a support vector machine (SVM), results in further classification accuracy gained over a Bayes' classifier.

Tan and Chen [15] proposed a novel approach of combining an unsupervised clustering scheme called AutoClass with Hidden Markov Models (HMMs) to determine the traffic density state in a Region Of Interest (ROI) of a road in a traffic video.

Maslekar et al. [3] claimed that clustering has demonstrated to be an effective concept to implement the estimation of vehicular density in the surroundings. However, due to high mobility, a stable cluster within a vehicular framework is difficult to implement. In this work, they proposed a direction based clustering algorithm with a clusterhead switching mechanism. The proposed algorithm facilitates the attaining of better stability, and thus improves the density estimation within the clusters.

All of these works established the importance of vehicular density awareness for neighboring areas, but none has deepened in the analysis of the accuracy of the method used to estimate this density, or the effect of the topology in the results obtained. Moreover, in most cases, this estimation does not take place in real time, or requires infrastructure deployment.

\section{CONCLUSIONS}

This paper proposes a method that allows vehicles to estimate the vehicular density in their neighborhood at any given time by using Vehicular Networks. Our proposal allows improving diffusion mechanisms to adapt the message dissemination policies to the specific traffic conditions.

To develop the vehicular density estimation algorithm, we have taken into account not only on the number of beacons received by vehicle, but also the topology of the map where the vehicles are located. As a result of a large number of simulations, using maps from different cities, we have obtained an equation that is able to accurately predict the vehicular density. Results show that our proposal allows estimating the vehicular density for any given city with a high accuracy, thereby allowing scientists to improve their proposals, or propose new solutions based on our findings.

\section{ACKNOWLEDGMENTS}

This work was partially supported by the Ministerio de Ciencia e Innovación, Spain, under Grant TIN2011-27543C03-01.

\section{REFERENCES}

[1] F. J. Martinez, J.-C. Cano, C. T. Calafate, P. Manzoni, and J. M. Barrios, "Assessing the feasibility of a VANET," in ACM Workshop on Performance Monitoring, Measurement and Evaluation of Heterogeneous Wireless and Wired Networks (PM2HW2N 2009, held with MSWiM). ACM New York, NY, USA, 2009, pp. 39-45, DOI: 10.1145/1641913.1641919.

[2] F. Soldo, R. Lo Cigno, and M. Gerla, "Cooperative synchronous broadcasting in infrastructure-to-vehicles networks," in Fifth Annual Conference on Wireless on Demand Network Systems and Services (WONS), Jan. 2008, pp. 125-132.

[3] N. Maslekar, M. Boussedjra, J. Mouzna, and H. Labiod, "A stable clustering algorithm for efficiency applications in VANETs," in 7th International Wireless Communications and Mobile Computing Conference (IWCMC), July 2011, pp. 1188-1193.

[4] F. J. Martinez, C. K. Toh, J.-C. Cano, C. T. Calafate, and P. Manzoni, "Determining the representative factors affecting warning message dissemination in VANETs," Wireless Personal Communications, pp. 1-20, 2011, DOI: 10.1007/s11277-011-0379-3.

[5] M. Fogue, P. Garrido, F. J. Martinez, J.-C. Cano, C. T. Calafate, and P. Manzoni, "Analysis of the most representative factors affecting Warning Message Dissemination in VANETs under real roadmaps," in 19th annual meeting of the IEEE International Symposium on Modeling, Analysis and Simulation of Computer and Telecommunication Systems (MASCOTS), Singapore, July 2011, pp. 197-204.

[6] K. Fall and K. Varadhan, "ns notes and documents," The VINT Project. UC Berkeley, LBL, USC/ISI, and Xerox PARC, February 2000, available at http://www.isi.edu/nsnam/ns/ns-documentation.html.

[7] Y.-C. Tseng, S.-Y. Ni, Y.-S. Chen, and J.-P. Sheu, "The broadcast storm problem in a mobile ad hoc network," Wireless Networks, vol. 8, pp. 153-167, 2002, DOI: 10.1023/A:1013763825347.

[8] M. Fogue, P. Garrido, F. J. Martinez, J.-C. Cano, C. T. Calafate, and P. Manzoni, "Evaluating the impact of a novel message dissemination scheme for vehicular networks using real maps," Transportation Research Part C: Emerging Technologies, vol. 25, pp. 61-80, December 2012, DOI: $10.1016 / j . t r c .2012 .04 .017$.

[9] F. Martinez, M. Fogue, C. K. Toh, J.-C. Cano, C. T. Calafate, and P. Manzoni, "Computer simulations of VANETs using realistic city topologies," Wireless Personal Communications, pp. 1-25, 2012. [Online]. Available: http://dx.doi.org/10.1007/s11277-012-0594-6

[10] S. Krauss, P. Wagner, and C. Gawron, "Metastable states in a microscopic model of traffic flow," Physical Review E, vol. 55, no. 5, pp. 5597-5602, 1997.

[11] M. Fogue, P. Garrido, F. J. Martinez, J.-C. Cano, C. T. Calafate, and P. Manzoni, "A Realistic Simulation Framework for Vehicular Networks," in 5th International ICST Conference on Simulation Tools and Techniques (SIMUTools 2012), Desenzano, Italy, March 2012, pp. 37-46.

[12] OpenStreetMap, "Collaborative project to create a free editable map of the world," 2012, available at http://www.openstreetmap.org.

[13] D. Krajzewicz and C. Rossel, "Simulation of Urban MObility (SUMO)," Centre for Applied Informatics (ZAIK) and the Institute of Transport Research at the German Aerospace Centre, 2012, Available at http://sumo.sourceforge.net/index.shtml.

[14] V. Tyagi, S. Kalyanaraman, and R. Krishnapuram, "Vehicular traffic density state estimation based on cumulative road acoustics," IEEE Transactions on Intelligent Transportation Systems, vol. PP, no. 99, pp. 1-11, 2012.

[15] E. Tan and J. Chen, "Vehicular traffic density estimation via statistical methods with automated state learning," in IEEE Conference on Advanced Video and Signal Based Surveillance (AVSS), Sept. 2007, pp. $164-169$. 